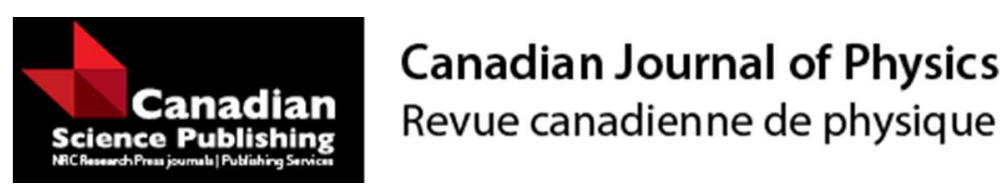

\title{
Solution of the Einstein-Maxwell Equations with Anisotropic Negative Pressure as a Potential Model of a Dark Energy Star
}

\begin{tabular}{|r|l|}
\hline Journal: & Canadian Journal of Physics \\
\hline Manuscript ID & cjp-2016-0069.R1 \\
\hline Manuscript Type: & Article \\
\hline Date Submitted by the Author: & $18-$ May-2016 \\
\hline Complete List of Authors: & $\begin{array}{l}\text { Bibi, Rashida; National University of Sciences, Mathematics } \\
\text { Feroze, Tooba; National University of Sciences, Mathematics } \\
\text { Siddiqui, Azad; National University of Sciences, Mathematics }\end{array}$ \\
\hline Keyword: & $\begin{array}{l}\text { Einstein-Maxwell Equations,, Anisotropic Pressure, Compact Stars, Exact } \\
\text { solutions, Relativistic objects }\end{array}$ \\
\hline
\end{tabular}

\section{SCHOLARONE ${ }^{\text {Tw }}$ \\ Manuscripts}




\title{
Solution of the Einstein-Maxwell Equations with Anisotropic Negative Pressure as a Potential Model of a Dark Energy Star
}

\author{
Rashida Bibi $^{1,2}$, Tooba Feroze ${ }^{1}$, and Azad A. Siddiqui ${ }^{1}$ \\ 1: School of Natural Sciences (SNS), \\ National University of Sciences and Technology (NUST), \\ H-12, Islamabad, Pakistan. \\ 2: Department of Mathematics and Statistics, \\ International Islamic University (IIU), \\ H-10, Islamabad, Pakistan. \\ E-mail: rashida.jahangir@iiu.edu.pk; teforze@sns.nust.edu.pk; azad@sns.nust.edu.pk
}

\begin{abstract}
We have obtained a new class of solutions for the Einstein-Maxwell field equations for static spherically symmetric spacetimes by considering the negative anisotropic pressures, which represents a potential model of a dark energy star. We take the equation of state $p_{r}=-\rho$, where $p_{r}$ is the radial pressure and $\rho$ is the density. We have also checked that for these solutions metric coefficients, mass density, radial pressure, transverse pressure, electric field, and current density are well defined for suitable values of the parameters involved in the solution. These exact solutions can be used to develop models of dark energy stellar interiors satisfying all physical constraints except for the causality condition, which cannot be satisfied for the equation of state considered here, and which is arguably not an applicable physical constraint for dark matter/energy.
\end{abstract}

PACS No: $04.20 \mathrm{jb}$

Keywords: Exact solutions, anisotropic pressure, dark energy

\section{Introduction}

The study of dark energy stars is of interest these days due to the fact that universe is expanding with acceleration which was suggested by High-z supernova Search Team in 1998 by observing type 1a supernova [1]. The acceleration of the universe can be explained by dark energy present in the universe which is about 26.7 percent of the total mass energy of the universe. Dark energy posses strong negative pressure which helps in explaining the acceleration of the expanding universe. The dark energy parameter is defined as $w=\frac{p_{r}}{\rho}$, where $p_{r}$ is the radial pressure and $\rho$ is the matter density. For accelerating expansion we have, $w<\frac{-1}{3}$ and $w=-1$ corresponds to the Einstein cosmological constant. In literature, a number of well behaved exact solutions of the Einstein-Maxwell field equations are obtained to model strange quark stars, neutron stars and pulsars for example in $[2,3]$. In this paper, we obtain a new solution of the Einstein-Maxwell field equations that corresponds to a dark energy star, by taking the equation of state as $p_{r}=-\rho$.

To model a relativistic stellar object, one generally assumes that the pressure distribution is isotropic and homogeneous for example in [4-7], but in the presence of the electromagnetic field pressure can be anisotropic. Anisotropy is defined as $p_{t}-p_{r}$, where $p_{t}$ is the transverse pressure. This represents an anisotropic force which is repulsive in nature if $p_{t}>p_{r}$ and attractive if $p_{t}<p_{r}$. Many compact astrophysical objects like X-ray pulsar, Her-X-1, X-ray buster $4 U 1820$ - 30, millisecond pulsar SAXJ1804.4 - 3658 etc have anisotropic pressures. Many solutions of the Einstein-Maxwell field equations have been obtained for anisotropic pressures. Thirukkanesh et al. [8, 9], Feroze [10], Mak et al. [11], Malaver [12, 13, 14, 15, 16], Sunzu et al. [17], Feroze et al. 
[18, 19], Maharaj et al. [20, 21], Takisa et al. [22] and Sharma et al. [23] obtained solutions of the Einstein-Maxwell field equations for charged static spherically symmetric spacetimes by assuming anisotropic pressure, in general, in all these solutions positive radial pressure is taken.

Mauz et al. [24, 25] obtained a solution for dark energy star named gravastar in which the interior of the star has de-Sitter spacetime with negative pressure $p=-\rho$. To get a stable star they assumed a thin shell of matter around the interior matter, having the equation of state $p=\rho$ and out side the star spacetime is the Schwarzschild spacetime. The solution is physically valid and stable. The solution of the gravastar is isotropic. Recently, Lobo [26] et al. have discussed the satiability of the thin shell gravastars and Ghosh et al. [27] have constructed higher dimensional charged gravastars.

In this paper, we obtain a class of physically valid solutions of the Einstein-Maxwell field equations having negative radial pressure and anisotropy. This represents model of the dark energy stars consisting solely of dark matter (i.e. with no ordinary hadronic matter admixed in). We have not assumed the de-Sitter spacetime in the interior of the dark energy star as chosen in [24] nor we assume some thin boundary around the star. In the Section 2, we discuss the Einstein-Maxwell field equations for the negative radial pressure by taking the equation of state as $p_{r}=-\rho$ and in Section 3 we obtain a class of solutions and discuss its validity.

\section{Field Equations For Charged Static Spherically Symmet- ric Spacetimes}

The Einstein-Maxwell field equations are given as

$$
\begin{aligned}
& T_{\mu \nu}=R_{\mu \nu}-\frac{1}{2} R g_{\mu \nu}, \\
&\left(\sqrt{-g}\left(F^{\mu \nu}\right)\right)_{, \nu}=j^{\nu} \sqrt{-g}
\end{aligned}
$$

where $T_{\mu \nu}$ is the stress energy tensor, $R_{\mu \nu}$ is the Ricci tensor, $R$ is the Ricci scalar, $g_{\mu \nu}$ is the metric tensor, $g$ is the determinate of metric tensor, $F^{\mu \nu}$ is the electromagnetic tensor, and $j^{\mu}$ is the current density.

For a solution of these field equations to be physically acceptable, as discussed in [28 - 30], the conditions are:

(1) There should no physical and geometric singularities in the solution.

(2) The density should be well defined, positive and a decreasing function of the radial parameter.

(3) The radial pressure should be positive and a decreasing function of radial parameter but for negative pressure this condition is not satisfied. Also at the boundary radial pressure should be zero, which is satisfied in case of negative pressure also.

(4) The solution should satisfy either weak energy condition or strong energy condition throughout within the star.

(5) The causality condition should be satisfied i.e. the speed of sound should be less than speed of light throughout the model i.e. $0<\frac{d p_{r}}{d \rho} \leq 1$. For dark energy case, this condition is not satisfied.

(6) The red shift should be positive, finite and monotonically decreasing in nature with the increase of $\mathrm{r}$.

(7) Electric field intensity $E$ should be well defined throughout within the domain of the solution.

(8) For the anisotropic case, the radial and the transverse pressures should be equal at the center i.e. $p_{r}(0)=p_{t}(0)$.

(9) The solution should match the Riessner-Nordstrom exterior solution at the boundary.

The metric for static spherically symmetric spacetimes can be given as

$$
d s^{2}=-e^{2 \nu(r)} d t^{2}+e^{2 \lambda(r)} d r^{2}+r^{2}\left(d \theta^{2}+\sin ^{2} \theta d \phi^{2}\right)
$$


The stress energy tensor for the metric in Eq.(3) for anisotropic matter is taken as [8]

$$
T_{\nu \mu}=\operatorname{diag}\left(-\rho-\frac{E^{2}}{2}, p_{r}-\frac{E^{2}}{2}, p_{t}+\frac{E^{2}}{2}, p_{t}+\frac{E^{2}}{2}\right),
$$

where $\rho$ is the density, $E$ is the electric field, $p_{r}$ is the radial pressure and $p_{t}$ is the transverse pressure. Here, magnetic field is taken to be zero and we only consider the electric field. For stress energy tensor given by Eq.(4), the Einstein-Maxwell field equations for the metric in Eq.(3) are

$$
\begin{aligned}
\frac{1}{r^{2}}\left(1-e^{-2 \lambda}\right)+\frac{2 \lambda^{\prime}}{r} e^{-2 \lambda} & =\rho+\frac{E^{2}}{2}, \\
-\frac{1}{r^{2}}\left(1-e^{-2 \lambda}\right)+\frac{2 v^{\prime}}{r} e^{-2 \lambda} & =p_{r}-\frac{E^{2}}{2}, \\
e^{-2 \lambda}\left(v^{\prime \prime}+v^{\prime 2}+\frac{v^{\prime}}{r}+v^{\prime} \lambda^{\prime}-\frac{\lambda^{\prime}}{r}\right) & =p_{t}+\frac{E^{2}}{2}, \\
\sigma & =\frac{1}{r^{2}} e^{-\lambda}\left(r^{2} E\right)^{\prime},
\end{aligned}
$$

where $\sigma$ is the current density. The trace $T$ of the stress energy tensor is

$$
T=e^{-2 \lambda}\left(2 \nu^{\prime \prime}+2 \nu^{\prime 2}-2 \lambda^{\prime} \nu^{\prime}+\frac{4}{r} \nu^{\prime}-\frac{4}{r} \lambda^{\prime}+\frac{2}{r^{2}}\right)-\frac{2}{r^{2}} .
$$

We assume following equation of state

$$
p_{r}=-\rho .
$$

Here, radial pressure is negative which corresponds to the dark energy. Using Eq. (10) in the Einstein-Maxwell field Eqs.(5) and (6), we have

$$
\nu=-\lambda,
$$

using Eqs. (10) and (11) in the Einstein-Maxwell field Eqs. (5)-(8), we get

$$
\begin{array}{r}
\frac{1}{r^{2}}\left(r\left(1-e^{-2 \lambda}\right)^{\prime}=\rho+\frac{E^{2}}{2},\right. \\
e^{-2 \lambda}\left(-\lambda^{\prime \prime}-\frac{2 \lambda^{\prime}}{r}\right)=p_{t}+\frac{E^{2}}{2}, \\
\sigma=\frac{1}{r^{2}} e^{-\lambda}\left(r^{2} E\right)^{\prime} .
\end{array}
$$

Using the transformation $Z=e^{-2 \lambda}$ in Eqs.(12)-(14), we finally have following set of three equations with five unknowns $Z, \rho, E, p_{t}$, and $\sigma$.

$$
\begin{array}{r}
1-Z-r Z^{\prime}=r^{2}\left(\rho+\frac{E^{2}}{2}\right), \\
r^{2} Z^{\prime \prime}+2 r Z^{\prime}=2 r^{2}\left(p_{t}+\frac{E^{2}}{2}\right), \\
\sigma=\frac{\sqrt{Z}}{r^{2}}\left(r^{2} E\right)^{\prime} .
\end{array}
$$

\section{A New Class of Solutions}

In order to obtain solution of the system of Eqs.(15)-(17), we assume the electric field, $E$ and the gravitational potential, $Z$ as

$$
E^{2}=\operatorname{Kar}^{2} \frac{5+a r^{2}}{\left(1+a r^{2}\right)^{3}}
$$




$$
Z=\frac{1}{\left(1+a r^{2}\right)}
$$

Using Eqs.(18) and (19) in Eqs.(15)-(17), the remaining unknowns are obtained as

$$
\begin{aligned}
\rho & =\frac{a\left(6+8 a r^{2}-5 k r^{2}+2 a^{2} r^{4}-a k r^{4}\right)}{2\left(1+a r^{2}\right)^{3}}, \\
p_{t} & =\frac{-a\left(6+5 k r^{2}+a r^{2}\left(-2+k r^{2}\right)\right)}{2\left(1+a r^{2}\right)^{3}} \\
\sigma & =\frac{\sqrt{a k}\left(15+4 a r^{2}+a^{2} r^{4}\right)}{\left(1+a r^{2}\right)^{3} \sqrt{5+a r^{2}}}
\end{aligned}
$$

and the metric (3) takes the form

$$
d s^{2}=-\frac{1}{1+a r^{2}} d t^{2}+\left(1+a r^{2}\right) d r^{2}+r^{2}\left(d \theta^{2}+\sin ^{2} \theta d \phi^{2}\right) .
$$

The solution obtained is physically acceptable as it satisfies the following conditions:

(1) There is no physical or geometrical singularity as $Z, E, \rho, p_{r}, p_{t}$, and $\sigma$ for $a>\frac{2}{3} k$ are well defined. (2) Density, $\rho$, is well defined for all $r$. Its value at the origin is $\rho(0)=3 a$, where $a>0$. From Eq.(20) it is clear that density is positive for all $r$ if $8 a-5 k>0$ and $2 a^{2}-a k>0$, which give $a>\frac{5 k}{8}$.

Density is a decreasing function of $r$. Taking derivative of Eq.(20) we have

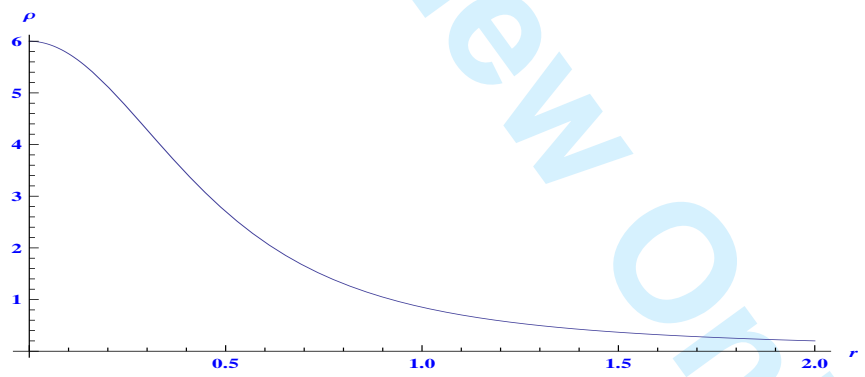

Figure 1: Density $\rho$ is plotted against the radial parameter $r$ by setting $a=2$ and $k=1$. From graph it is clear that density is well defined, positive and a decreasing function of $r$.

$$
\frac{d \rho}{d r}=\frac{-a r\left(5 k+10 a+r^{2}\left(12 a^{2}-8 a k\right)+r^{4}\left(2 a^{3}-k a^{2}\right)\right.}{\left(1+a r^{2}\right)^{4}} .
$$

We can set suitable values of parameters $a$ and $k$ so that $d \rho / d r<0$, i.e. $\rho$ becomes monotonically decreasing with respect to $r$. If we set $12 a^{2}-8 a k>0$ and $2 a^{3}-k a^{2}>0$ then we have $a>\frac{2}{3} k$. For this the density becomes monotonically decreasing. But it is positive definite if $a>\frac{5 k}{8}$, so we can say that the density is positive definite and monotonically decreasing if $a>\frac{2 k}{3}$. For example if we choose $a=1$ and $k=1$ we get $\rho>0$ and $d \rho / d r<0$ which is also shown in Fig. 1.

(3) From Eq.(10) it is clear that the radial pressure is negative and not a decreasing function of the radial parameter. To require that the radial pressure is zero at the boundary, $r=R$, we have

$$
6+8 a R^{2}-5 k R^{2}+2 a^{2} R^{4}-a k R^{4}=0 .
$$

(4) As $\rho>0$ within the star so solution satisfies the weak energy condition.

(5) From Eq.(10) $d p_{r} / d \rho=-1$, so the causality condition is not satisfied as mentioned in Section2. 


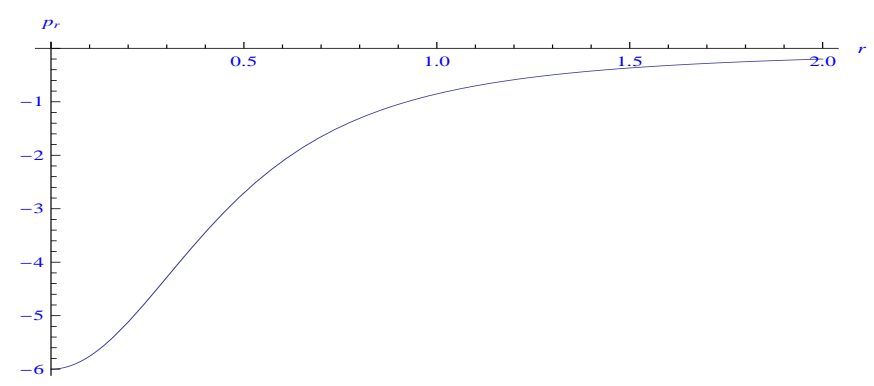

Figure 2: Radial pressure $p_{r}$ is plotted against the radial parameter $r$ by setting $a=2$ and $k=1$. Radial pressure is well defined and at the boundary $r=R$ its value is zero.

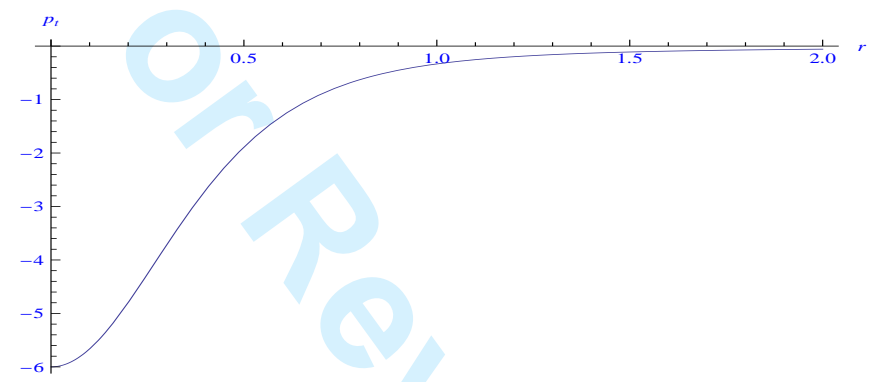

Figure 3: Transverse pressure $p_{t}$ is plotted against the radial parameter $r$ by setting $a=2$ and $k=1$. Transverse pressure is well defined for all $r$.

(6) The red shift at the origin $Z(0)$ and on the boundary $Z(R)$ are finite and positive. From Eq.(19), we get $Z(0)=1>0$ and $Z(R)=\frac{1}{1+a R^{2}}>0(a>0)$ where $r=R$ is the boundary.

(7) From Eq.(18) it is clear that the electrical field is well defined within the star.

(8) Radial and transverse pressures are same at the origin. Eq.(10) with Eq.(20) and Eq.(21) give $p_{r}(0)=p_{t}(0)=-3 a$. Figs. 2 and 3 show the radial and the transverse pressures respectively for $a=2$.

(9) The requirement that the solution matches the Riessner-Nordstrom exterior solution at the boundary, with $g_{00}=1-\frac{2 M}{R}+\frac{Q^{2}}{R^{2}}$ and $E(R)=\frac{Q}{R^{2}}$, gives

$$
\begin{aligned}
\frac{1}{1+a R^{2}} & =1-\frac{2 M}{R}+\frac{Q^{2}}{R^{2}}, \\
\frac{Q^{2}}{R^{6}} & =\frac{a k\left(5+a R^{2}\right)}{\left(1+a R^{2}\right)^{3}},
\end{aligned}
$$

where $M$ is the total mass of the star. Using Eq.(27) and $a>\frac{2 k}{3}$, Eq.(26) gives

$$
\frac{M}{R}<\frac{a R^{2}\left(2+5 a^{2} R^{4}+19 a R^{2}\right)}{4\left(1+a R^{2}\right)^{3}}
$$

which gives bound on the the mass-radius ratio.

Also using Eq.(27) and $a>\frac{2 k}{3}$ we get the bound on the charge-radius ratio as

$$
\frac{Q^{2}}{R^{2}}<\frac{3 a^{2} R^{4}\left(5+a R^{2}\right)}{2\left(1+a R^{2}\right)^{3}} .
$$




\section{Stability}

Stability of an ordinary star depends on the behavior of radial sound velocity $V_{r}^{2}=\frac{d p_{r}}{d \rho}$ and transverse sound velocity $V_{t}^{2}=\frac{d p_{t}}{d \rho}$. The region in which, the radial sound velocity is greater than the transverse sound velocity is called stable region [31,32]. As in Section 2 it is mentioned that the dark energy stars with equation of state $p_{r}=-\rho$ do not satisfy the causality condition, however, we can check the stability region. The radial sound velocity and the transverse sound velocity are

$$
\begin{aligned}
\frac{d p_{r}}{d \rho} & =-1, \\
\frac{d p_{t}}{d \rho} & =\frac{\left(-5 k+a^{2} r^{2}\left(-4+k r^{2}\right)+4 a\left(5+2 k r^{2}\right)\right)}{\left(-5 k-2 a^{3} r^{4}+a^{2} r^{2}\left(-12+k r^{2}\right)+2 a\left(-5+4 k r^{2}\right)\right)}
\end{aligned}
$$

and the difference is

$$
\frac{d p_{r}}{d \rho}-\frac{d p_{t}}{d \rho}=-1-\frac{\left(-5 k+a^{2} r^{2}\left(-4+k r^{2}\right)+4 a\left(5+2 k r^{2}\right)\right)}{\left(-5 k-2 a^{3} r^{4}+a^{2} r^{2}\left(-12+k r^{2}\right)+2 a\left(-5+4 k r^{2}\right)\right)} .
$$

From Eq.(32) it is clear that we can set $\frac{d p_{r}}{d \rho}-\frac{d p_{t}}{d \rho}>0$ by choosing suitable values of the parameters $a$ and $k$. For example the difference of radial and transverse sound velocities is plotted for different values of the parameter $a$ and $k$ and this difference is positive for some region which can be seen in the Fig. 4.

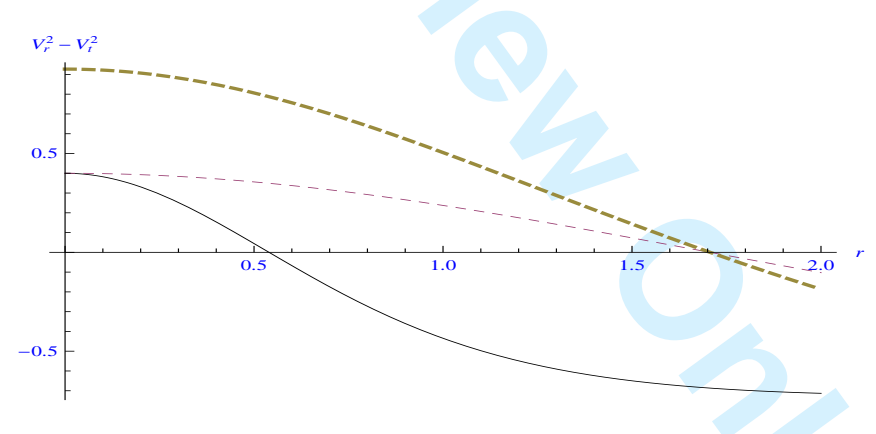

Figure 4: Difference of the radial sound velocity and the transverse sound velocity is plotted against the radial parameter $r$ for different values of parameters. The solid curve is for $a=2$ and $k=1$, the dashed curve is for $a=0.2$ and $k=0.1$ and the bold-dashed curve is for $a=0.2$ and $k=0.01$.

\section{Conclusion}

We have found a physically valid class of solutions which represents a model for dark energy stars. The radial and transverse pressures, density, electrical field and all coefficients of the metric are well defined and there is no singularity present. The solution obtained fulfills all the requirements of a compact negative energy stellar object, however, our solution does not satisfy the causality condition. Ellis at al. (2007) have discussed violation of this condition for different theories. For dark energy case, Das and Ali (2014) have dealt with this issue by admixing the ordinary hadronic matter. It would be worthwhile to follow the similar procedure and modify our solution so that causality condition is also satisfied. 


\section{Acknowledgements}

Authors are grateful to the unknown referee for his valuable comments/suggestions.

\section{References}

[1] Filippenko AV, Riess AG. 1998. Physics Reports 307: 31.

[2] Pant N, Negi PS. 2012. Astrophysics and Space Science 338: 157.

[3] Pant N, Pradhan N, Bansal RK. 2016. Astrophysics and Space Science 361: 1.

[4] Kumar J, Gupta YK. 2014. Astrophysics and Space Science 351: 243.

[5] Mahbubur Rahman AHM, Murad MH. 2014. Astrophysics and Space Science 351: 255.

[6] Pant N, Pradhan N, Murad MH. 2014. Astrophysics and Space Science 352: 135.

[7] Pradhan N, Pant N. 2014. Astrophysics and Space Science 352: 143.

[8] Thirukkanesh S, Maharaj SD. 2008. Classical and Quantum Gravity 25: 235001.

[9] Thirukkanesh S, Ragel FC. 2014. Pramana Journal of Physics 83: 83.

[10] Feroze T. 2012. Canadian Journal of Physics 90: 1179.

[11] Mak MK, Harko T. 2004. International Journal of Modern Physics D 13: 1.

[12] Malaver M. 2015. International Journal of Astrophysics and Space Science 3: 6.

[13] Malaver M. 2014. Frontiers of Mathematics and its Applications 1: 9.

[14] Malaver M. 2015. AASCIT Journal of Physics 1: 104.

[15] Malaver M. 2013. American Journal of Astronomy and Astrophysics 1:41 .

[16] Malaver M. 2013. World Applied Programming 3: 309.

[17] Sunzu JM, Maharaj SD, Ray S. 2014. Astrophysics and Space Science 354: 517.

[18] Feroze T, Siddiqui AA. 2014. Journal of the Korean Physical Society 65: 944.

[19] Feroze T, Siddiqui AA. 2011. General Relativity and Gravitation 43: 1025.

[20] Maharaj SD, Takisa PM. 2012. General Relativity and Gravitation 44: 1419.

[21] Maharaj SD, Takisa PM. 2013. General Relativity and Gravitation 45: 1951.

[22] Takisa PM, Maharaj SD, Ray S. 2014. Astrophysics and Space Science 354: 463.

[23] Shrama R, Ratanpal BS. 2013. International Journal of Modern Physics D 22: 1350074.

[24] Mazur P, Mottola E. 2001. arXiv:gr-qc/0109035, Report number: LA-UR-01-5067.

[25] Mazur P, Mottola E. 2004. Proceedings of the National Academy of Sciences USA 101: 9545.

[26] F. S. N. Lobo FSN, Martn-Moruno P, Montelongo-Garca N, Visser M. 2015. The Fourteenth Marcel Grossmann Meeting on General Relativity, University of Rome "La Sapienza".

[27] Ghosh S, Rahaman F, Guha BK, Ray S. 2015. arXiv:1511.05417. 
[28] Gupta YK, Maurya SK. 2011. Astrophysics and Space Science 332: 155.

[29] Pant N, Mehta RN, Pant MJ. 2010. Astrophysics and Space Science 330: 353.

[30] Pant N, Mehta RN, Pant MJ. 2011. Astrophysics and Space Science 332: 473.

[31] Herrera L. 1992. Physical Letter A 206: 185.

[32] Maurya SK, Jasim MK, Gupta YK, Smitha TT. 2016. Astrophysics and Space Science 361: 1. 